Research Article

\title{
Comparative Analysis of Soil-Water Characteristic Curve in Fractal and Empirical Models
}

\author{
Gaoliang Tao $\mathbb{D},{ }^{1,2}$ Yangyang Chen, ${ }^{1}$ Henglin Xiao $\mathbb{D D}^{1},{ }^{1}$ Yin Chen $\mathbb{D}^{3},{ }^{3}$ and Wan Peng $\mathbb{D}^{1}$ \\ ${ }^{1}$ School of Civil, Architectural \& Environmental Engineering, Hubei University of Technology, Wuhan 430068, China \\ ${ }^{2}$ School of Urban Construction, Wuchang University of Technology, Wuhan 430223, China \\ ${ }^{3}$ Institute of Geophysics \& Geomatics, China University of Geosciences, Wuhan 430074, China \\ Correspondence should be addressed to Henglin Xiao; xiao-henglin@163.com and Yin Chen; agchen19930922@163.com
}

Received 23 December 2019; Revised 24 March 2020; Accepted 15 April 2020; Published 5 June 2020

Academic Editor: Francesco Ruffino

Copyright (C) 2020 Gaoliang Tao et al. This is an open access article distributed under the Creative Commons Attribution License, which permits unrestricted use, distribution, and reproduction in any medium, provided the original work is properly cited.

The fitting analysis of soil-water characteristic curve (SWCC) is the foundation of engineering properties of unsaturated soils. The purpose of this paper is to explore the differences of fitting effects between different fractal and empirical models as well as the effect of residual water content $\theta_{r}$ on the model fitting. The experimental SWCCs of Hunan clay at different initial dry densities are measured by pressure plate instrument, and then they are fitted by fractal and empirical models. The effect of the limit of $\theta_{r}$ on the fitting results is analyzed by comparing the results of indoor evaporation test. The results show that within the measured data point range, fractal model II-2 and van Genuchten model (VG model) have the best fitting effect, fractal models I and II-1 take the second place, and the fitting effect of Gardner model is the worst. When the matrix suction is more than the measured data, the fitting accuracy of the model is poor. For the model with $\theta_{r}$, reasonable limit range of $\theta_{r}$ can only improve the fitting accuracy of the model to a certain extent. For the fractal model, the fitting accuracy of the model can be improved effectively when $\theta_{r}$ is considered. Furthermore, the difference of fractal dimension and air-entry value in various fractal models are also discussed in this paper.

\section{Introduction}

The soil-water characteristic curve (SWCC) plays an important role in the study of the hydraulic characteristics of unsaturated soils, and it is a significant foundation in the field of predicting the strength, permeability, constitutive relation, and consolidation theory of unsaturated soils [1-5].

Generally, the data obtained from SWCC experiment is a series of discrete points. In order to investigate the relationship between SWCC and the physical properties of soil structure stability, particle size distribution, and water holding capacity, it is necessary to fit the smooth curve through various models. Therefore, it is a core problem to adopt the appropriate SWCC model. Since the 1950s, Gardner [6], van Genuchten [7], and Fredlund and Xing [8] proposed some empirical models to quantitatively describe the soil-water relationship. From then on, a large number of researchers have further established various models to describe SWCC more accurately. For example, Gallipoli et al.
[9] proposed a SWCC expression considering the change of porosity, which was capable of describing the volume change of soil induced by stress state. Pham et al. [10] established a SWCC model considering hysteretic effect by ignoring the volume change of soil. Omuto [11] proposed a double-index SWCC model through analyzing the soil structure and pore spatial structure. Based on van Genuchten model (VG model), Zhang et al. [12] further presented a SWCC model taking the change of void ratio into account. Ahangar-Asr et al. [13] presented a SWCC model from evolutionary polynomial regression (EPR) technology, and then the accuracy was validated by using the experimental data of various soils. Huang et al. [14] proposed a SWCC model considering soil deformation and hysteresis effect, by comparing some classical SWCC models. Wang et al. [15] further proposed a double stress generalized SWCC model considering soil deformation and pore size distribution. Pham and Fredlund [16] developed a gravimetric SWCC equation considering volume changes and further utilized 
the model to fit the soil suction-gravimetric water content data of different soils. Roy and Rajesh [17] measured suction-water content data of slurry and compacted soils from tensiometers and dew point potentiometer. Further, five classical SWCC models were used to fit and evaluate the significant parameters of different models in the full suction range. Ren et al. [18] proposed a new approach to estimate entire SWCC from the characteristic of the shape of experimental SWCC, and the application of this approach on the coarse-grained and fine-grained soil was verified. Zhai et al. [19] demonstrated that the residual water content has great influence on the fitting equation through measured experimental SWCCs of different soils. Rajesh et al. [20] adopted five existing SWCC models to fit the laboratory data using weighted method of least squares, indicating that the fitting results have great dependency on the parameter of model.

Out of various SWCC models available in the literature, classical models such as the Gardner model, the van Genuchten (VG) model, and the Fredlund-Xing (FX) model are often widely used by researchers. For example, Puppala et al. [21] adopted FX model to fit SWCCs of expansive soils and then obtained the relationship between parameters of model and physical properties of soil. Kim and Borden [22] determined shear strength of different soils by using FX model and then investigated the effect of stress state on the shear strength. Chen and Gong [23] compared the fitting effects of five SWCC models, indicating that the modified Gardner model has the best performance. Zhang et al. [24] found that both VG model and FX model can accurately describe SWCC of Jiaohe soil over the complete range of matrix suction. The above models are in the simple from and are convenient to implement. However, most of them belong to empirical equations, so many parameters in them have no clear physical significance. Meanwhile, their fitting results depend on the limit range of parameters, resulting in that the fitting results have multisolution.

The above models are comparatively simpler to use and convenient to implement in establishing a smooth SWCC. However, most of them contain empirical equations, so many parameters in them have no clear physical significance. Their fitting results are also dependent on the limiting range of the parameters utilized, resulting in multiple solutions. Since Mandelbrot [25] proposed fractal theory, it has been widely applied in practical engineering problems [26-30]. It has been reported that SWCC is closely related to soil structure, such as surface area, volume, and size distribution of particle or pore, which present self-similar characteristics. Therefore, fractal theory has been recognized as a useful tool to investigate SWCC [25, 31-33]. Tyler and Wheatcraft [34] derived a fractal SWCC model based on Sierpinski's model to simulate pore fractal dimension. Rieu and Sposito [35] derived a fractal SWCC model in terms of mass distribution based on Menger's sponge model. On this basis, Perfect et al. [36] proposed a new SWCC model. Perrier et al. $[37,38]$ presented a pore-solid fractal model to simulate soil structure. $\mathrm{Xu}$ et al. [39, 40] derived a general SWCC expression through cumulative number of pores and the pore size and then divided the existing fractal models into three categories. Crawford et al. [41] established a SWCC model with a fractal dimension of pore surface. Tao et al. [42] established a fractal model to describe the influence of clay content on SWCC and determined the variation of clay content with fractal dimension. Yang et al. [43] proposed a SWCC equation considering hysteresis effect by analyzing the fractal characteristics of the pore size and the seepage path of unsaturated soils from microscopic perspective.

Fitting SWCC is an important foundation to study the hydraulic characteristics of unsaturated soils, and the accuracy of model fitting has a great influence on the study of geotechnical engineering properties of unsaturated soils. However, the existing SWCC fitting results reveal that the fitting results of different models for the same experimental data are significantly different, indicating that it is necessary to elaborate the fitting characteristics of different models. In addition, most existing fitting studies are based on empirical models, and there are few studies on fitting fractal models. Compared with empirical model, fractal model has fewer parameters with obvious physical meaning and has simple fitting process. Therefore, fitting SWCC by fractal model is a method worthy of in-depth study. This paper summarizes the existing SWCC fractal models and divides it into two categories according to the different derivation process. Based on the obtained experimental data of the pressure plate instrument, the fitting effects of two types of fractal models and existing empirical models (Gardner and VG model) are analyzed.

\section{Soil-Water Characteristic Curve Model}

\subsection{SWCC Fractal Model}

2.1.1. Fractal Model I. Zhang et al. [44] proposed a porosity model, which is expressed as

$$
\phi(>r)=1-\left(\frac{r}{r_{\max }}\right)^{3-D}
$$

where $\phi$ is the soil porosity, $r$ is the pore size of soil, $r_{\max }$ is the maximum pore size, and $D$ is the fractal dimension.

Taking $r$ as the boundary condition, if the pores of unsaturated soils with pore size less than or equal to $r$ are filled with water, then this can be expressed as

$$
\theta_{s}=\phi(>r)+\theta
$$

where $\theta_{s}$ is the saturated soil volumetric water content and $\theta$ is the volumetric water content.

Coupling (1) with (2) gives

$$
\theta_{s}=\theta+1-\left(\frac{r}{r_{\max }}\right)^{3-D} \text {. }
$$


According to Young-Laplace equation, the relationship between matrix suction $\psi$ and pore sizes $r$ can be expressed as follows:

$$
\psi=\frac{2 T_{s} \cos \alpha}{r}
$$

where $\psi$ is the matrix suction ( $\psi$ in all equations is specified such that $\psi>0$ ) and $\alpha$ is the contact angle. In the constant temperature condition, $2 T_{s} \cos \alpha$ can be assumed as a constant.

Substituting (4) into (3) yields the following expression:

$$
\theta_{s}=\theta+1-\left(\frac{\psi}{\psi_{a}}\right)^{D-3},
$$

where $\psi_{a}$ is the air-entry value.

Further, (5) can be represented as

$$
\theta=\left(\frac{\psi}{\psi_{a}}\right)^{D-3}-\frac{1}{e+1},
$$

where $e$ refers to the void ratio.

It should be noted that (6) is effective only in the range of $\psi>\psi_{a}$. If matrix suction $\psi$ is less than $\psi_{a}$, the soil sample is assumed to be fully saturated. Then, the fractal model for SWCC model is written as

$$
\begin{cases}\theta=\left(\frac{\psi}{\psi_{a}}\right)^{D-3}-\frac{1}{e+1}, & \psi \geq \psi_{a} \\ \theta=\frac{e}{e+1}, & \psi<\psi_{a} .\end{cases}
$$

\subsubsection{Fractal Model II (1) Fractal Model II-1}

Another porosity model presented by Zhang et al. [44] is expressed as follows:

$$
\phi(>r)=\left(\frac{r_{\max }}{L}\right)^{3-D}-\left(\frac{r}{L}\right)^{3-D},
$$

where $L$ is the total observation scale of soil.

Coupling (8) with (2) gives

$$
\theta_{s}=\theta+\left(\frac{r_{\max }}{L}\right)^{3-D}-\left(\frac{r}{L}\right)^{3-D} \text {. }
$$

It is assumed that $A$ is equal to $\left(r_{\max } / L\right)^{3-D}$. Then, combining (9) with (4) yields

$$
\theta_{s}=\theta+A\left[1-\left(\frac{\psi}{\psi_{a}}\right)^{D-3}\right] \text {. }
$$
as

When $r$ approaches $0, \theta_{s}=A$; then (10) can be rewritten

$$
\frac{\theta}{\theta_{S}}=\left(\frac{\psi}{\psi_{a}}\right)^{D-3}
$$

It can be rewritten as, (11)

$$
\theta=\left(\frac{\psi}{\psi_{a}}\right)^{D-3} \frac{e}{e+1} .
$$

Note that (12) is only valid only in the range of $\psi>\psi_{a}$. If matrix suction $\psi$ is less than $\psi_{a}$, the soil sample is assumed to be fully saturated. Then, the fractal SWCC model is expressed as follows:

$$
\begin{cases}\theta=\left(\frac{\psi}{\psi_{a}}\right)^{D-3} \frac{e}{e+1}, & \psi \geq \psi_{a}, \\ \theta=\frac{e}{e+1}, & \psi<\psi_{a} .\end{cases}
$$

(2) Fractal Model II-2

When the residual water content is considered, the tiny pores corresponding to the residual water content are regarded as the components of particles; then (11) becomes

$$
S_{e}=\left(\frac{\psi}{\psi_{a}}\right)^{D-3},
$$

where $S_{e}$ is the effective degree of saturation.

The dimensionless water content variable $\Theta$ is introduced here, which can be expressed as standardized volumetric water content by saturated water content and residual water content:

$$
\Theta=S_{e}=\frac{\theta-\theta_{r}}{\theta_{s}-\theta_{r}},
$$

where $\theta_{r}$ is the residual water content.

Substituting (15) into (14) gives

$$
\theta=\left(\frac{\psi}{\psi_{a}}\right)^{D-3}\left(\frac{e}{e+1}-\theta_{r}\right)+\theta_{r}
$$

Note that (16) is only valid in the range of $\psi>\psi_{a}$. If matrix suction $\psi$ is less than $\psi_{a}$, the soil sample is assumed to be fully saturated. Then, the fractal SWCC model is expressed as follows:

$$
\begin{cases}\theta=\left(\frac{\psi}{\psi_{a}}\right)^{D-3}\left(\frac{e}{e+1}-\theta_{r}\right)+\theta_{r}, & \psi \geq \psi_{a} \\ \theta=\frac{e}{e+1}, & \psi<\psi_{a}\end{cases}
$$

2.2. Empirical Model. Gardner [6] proposed a SWCC model with two parameters, which is expressed as

$$
\theta=\theta_{r}+\frac{\theta_{s}-\theta_{r}}{1+\lambda \psi^{n}},
$$

where $\lambda$ is a parameter related to the air-entry value and $n$ is a parameter related to the drying rate.

van Genuchten [7] presented a SWCC model with three parameters, and its expression is as follows: 
TABLE 1: Basic physical parameters of Hunan clay.

\begin{tabular}{lcc}
\hline Relative density & Liquid limit (\%) & Plastic limit (\%) \\
\hline 2.76 & 46.34 & 27.84 \\
\hline
\end{tabular}

$$
\theta=\theta_{r}+\frac{\theta_{s}-\theta_{r}}{\left[1+(a \psi)^{m}\right]^{p}}
$$

where $a, m$, and $p$ are fitting parameters, $a$ is related to the air-entry value, $m$ is related to the pore size distribution of soil, and $p$ is the parameter related to $m$ as $p=1-1 / m$.

\section{Materials and Methods}

3.1. Experiment Materials. Taking Hunan clay as the studied object, its basic physical index is shown in Table 1 . According to the plastic soil classification in standard for soil test method, the experimental soil belongs to the lowplasticity clay. It has the characteristics of high clay content, high liquid limit, high saturation, and low permeability coefficient.

\subsection{SWCC Test}

3.2.1. Experiment Methods and Processes. Pressure plate tests were performed to measure the SWCCs of Hunan clay, where the pressure plate instrument is manufactured by the American Soilmoisture Company. The pressure plate instrument used in the experiment, which consists of pressure cell, 15-bar air-entry value (HAE) ceramic disc, pressure gauge, and nitrogen source, is shown in Figure 1.

Before the experiment, the soil was dried, then crushed, and screened by a $2 \mathrm{~mm}$ sieve. Then, some distilled water was uniformly sprayed on the dried soil so that the initial water content of the soil is about 19\% which is close to the optimum water content. Subsequently, the soil was put in a sealed box for at least 3 days to make water distribute uniformly. After the required time, samples with six different initial dry densities were prepared by hydraulic jack in a circular mold of $61.8 \mathrm{~mm}$ inner diameter and $20 \mathrm{~mm}$ height, respectively. The dry density $\rho_{d}$ was $1.30 \mathrm{~g} / \mathrm{cm}^{3}, 1.35 \mathrm{~g} / \mathrm{cm}^{3}$, $1.40 \mathrm{~g} / \mathrm{cm}^{3}, \quad 1.45 \mathrm{~g} / \mathrm{cm}^{3}, \quad 1.50 \mathrm{~g} / \mathrm{cm}^{3}$, and $1.60 \mathrm{~g} / \mathrm{cm}^{3}$, respectively.

The specific test steps are as follows: (1) The ring knife sample with different dry densities, together with the HAE ceramic disc, was saturated. (2) The specimen, together with the stainless steel cutting rings, was placed on the HAE ceramic disc in the pressure cell. (3) The applied air pressure was imposed on the specimen when the pressure cell was sealed. (4) The water drained from the samples was recorded during the whole process of the test. When the sample stops draining, the current suction level was considered to be balanced. Then, the drainage valve was closed and the applied air pressure was released. Meanwhile, it was necessary to remeasure the weight of the sample and calculate the corresponding water content. (5) The above steps were repeated to set the next suction level.
3.2.2. Test Results and Analysis. Figure 2 shows the experimental SWCCs in the form of volumetric water content. Obviously, water content decreases with the increase of matrix suction, and the water content corresponding to matrix suction less than $0.1 \mathrm{kPa}$ is equivalent to the saturated water content. For the stage of matrix suction is less than $5 \mathrm{kPa}$, the larger the initial dry density is, the smaller the change of volumetric water content is. When the matrix suction is in the range of $5 \sim 80 \mathrm{kPa}$, the change rate of volumetric water content is the largest. In addition, when the matrix suction reaches $80 \mathrm{kPa}$, the volumetric water content changes linearly with the matrix suction, and the dehydration rates of different dry densities are approximately equal. A summary of data used is provided in Supplementary Table S1.

\subsection{Indoor Evaporation Experiment and Determination of} Residual Water Content. For the preparation of experiment, a set of parallel samples was prepared the same as the pressure plate instrument test. And then, they were placed in laboratory (indoor temperature is about $24^{\circ} \mathrm{C}$ ). In natural state, the samples were subjected to double-sided exposure and air-drying. The specific test procedure is as follows: (1) The sample with different dry densities was saturated. (2) The weights of sample were measured at intervals, and the water content of the sample was calculated. (3) The experiment was terminated until the mass of sample remained unchangeable. Taking the mass water content as the longitudinal coordinate and the time $H$ as transverse coordinate, the variation curve of mass water content with time is drawn, as shown in Figure 3. A summary of data used is provided in Supplementary Table S2.

Macroscopically, water evaporation in soil refers to the dynamic process where water changes from liquid state to gas state, which can be divided into three stages: (1) stable stage, (2) deceleration stage, and (3) residual stage. It can be seen from Figure 3 that little water can be evaporated as the evaporation time gradually lengthened. When the evaporation time reached $80 \mathrm{~h}$, the mass water content of the six samples has little difference, indicating that the evaporation behavior of soil water was finished, namely, residual stage. Due to the adsorption of soil particles, part of the bound water will remain in the soil after drying process, which contributes to the existence of residual water content $\theta_{r}$. The values of $\theta_{r}$ of six samples with different initial dry densities are shown in Table 2.

\section{Model Fitting}

In recent years, many scholars have done a lot of research on SWCC and put forward many effective prediction models and used the model to predict the SWCC. However, the fitting results of different models of the same soil are quite different, and most of the existing researches are dominated by empirical models and lack of research on fractal model fitting. In addition, the fitting characteristics of empirical model and fractal model have not been explained. In this 


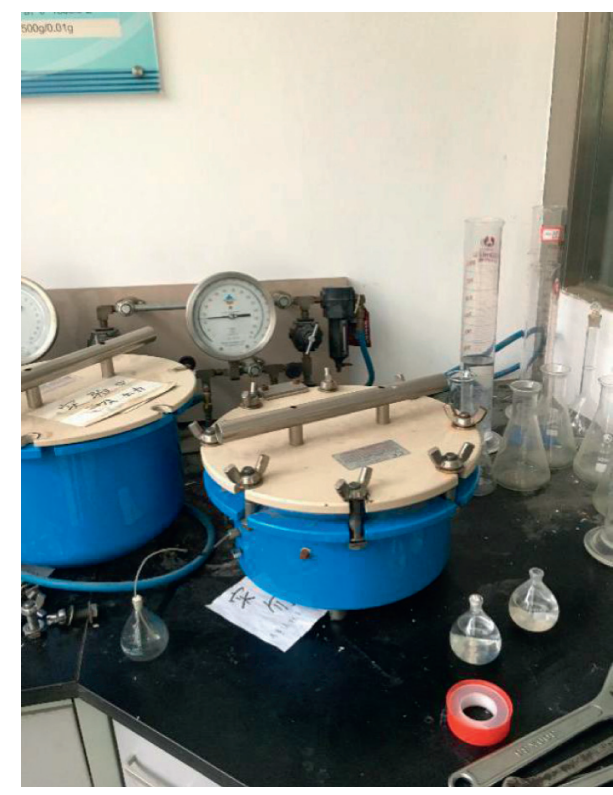

(a)

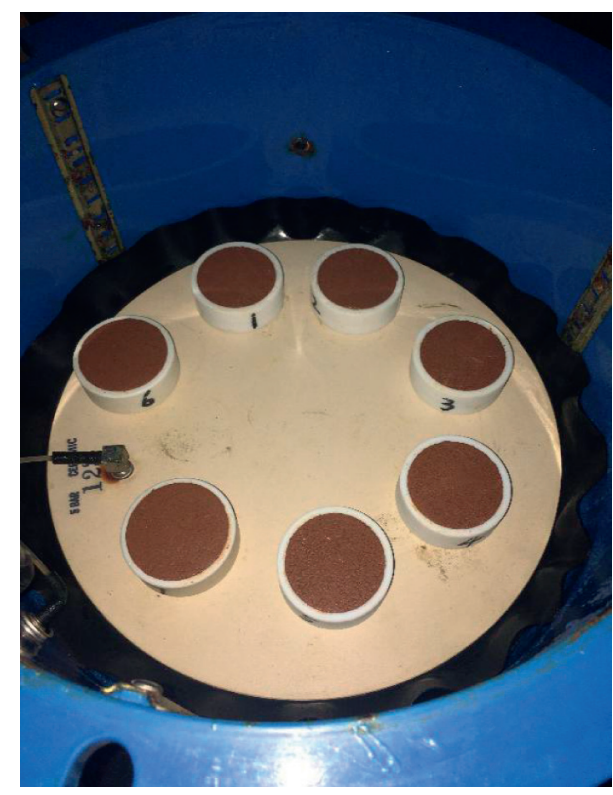

(b)

Figure 1: Test steps for pressure plate instrument.

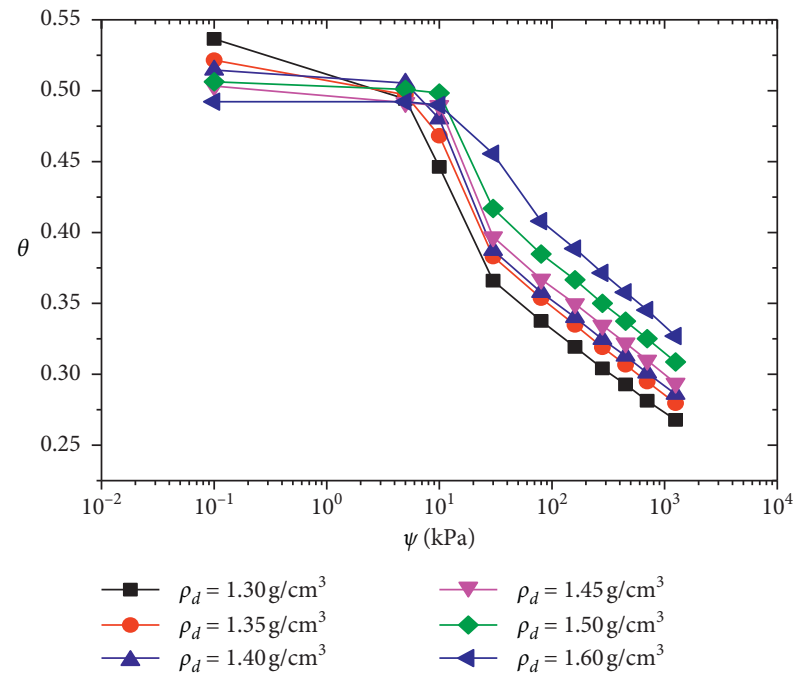

FIGURE 2: Measured values of SWCCs of Hunan clay with different initial dry densities.

paper, based on experimental SWCCs, the fitting results of different models are compared by MATLAB software, and a suitable model is suggested to fit experimental SWCCs measured by pressure plate instrument. In addition, when the model includes parameter $\theta_{r}$, the influence of the limited range of $\theta_{r}$ on the model fitting is analyzed from the data of indoor evaporation test.

4.1. The Fitting Analysis and Comparison of Different Models. MATLAB software is exploited to fit SWCC by two-category fractal model, Gardner model and VG model, respectively.

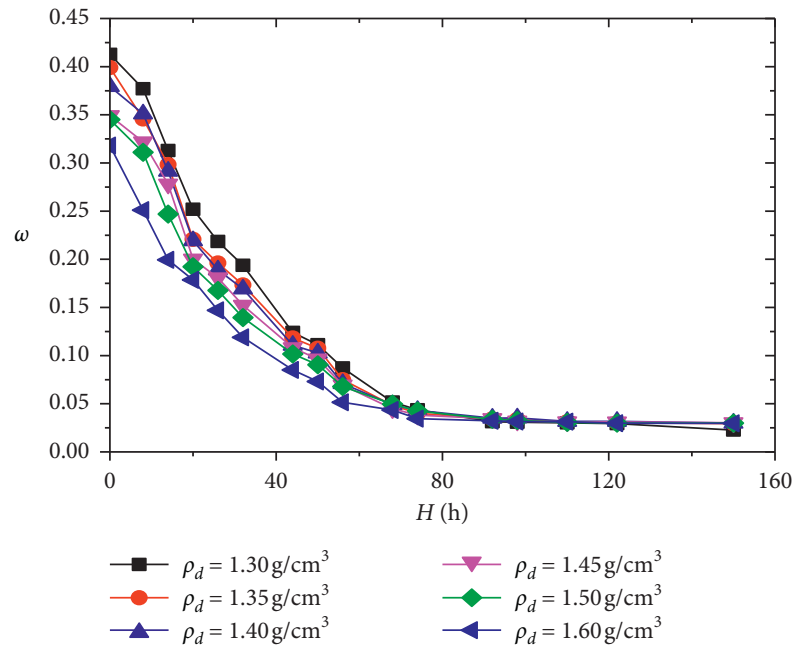

Figure 3: Variation curve of mass water content of Hunan clay with time.

TABLE 2: Residual water content determined by evaporation test.

\begin{tabular}{lllllll}
\hline Initial dry density $\left(\mathrm{g} / \mathrm{cm}^{3}\right)$ & 1.30 & 1.35 & 1.40 & 1.45 & 1.50 & 1.60 \\
\hline Mass water content, $\omega_{r}(\%)$ & 2.27 & 2.94 & 2.99 & 3.02 & 2.99 & 2.96 \\
Volumetric water content, & 2.95 & 3.97 & 4.17 & 4.38 & 4.45 & 4.74 \\
$\theta_{r}(\%)$ & & & & & &
\end{tabular}

Observing the results of indoor evaporation test, the values of $\theta_{r}$ of six samples are found to be less than $5 \%$. For the model with parameters $\theta_{r}$, the range of $\theta_{r}$ is limited to $0<\theta_{r} \leq 5 \%$. The fitting results of different models are shown in Figure 4; it is found that within the measured data point range, the fractal model II-2 and the VG model have the best fitting effect, fractal models I and II-1 take the second place, and the fitting effect of the Gardner model is the worst. It 


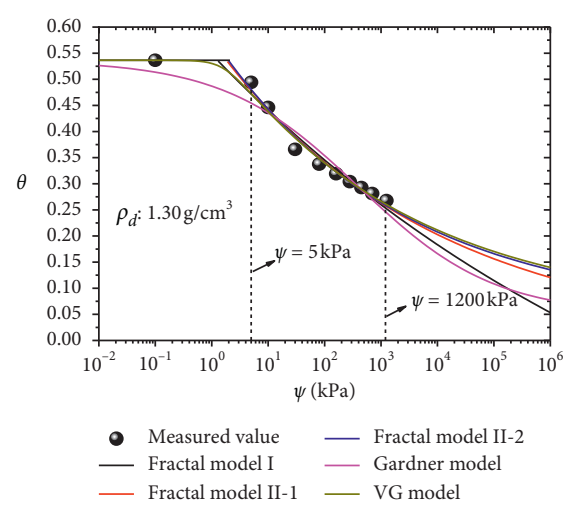

(a)

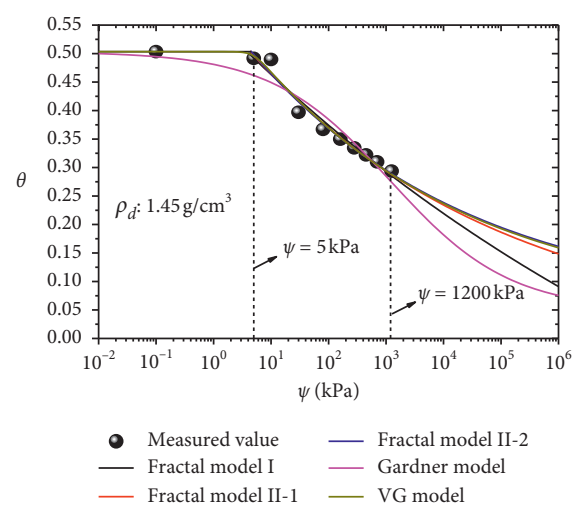

(d)

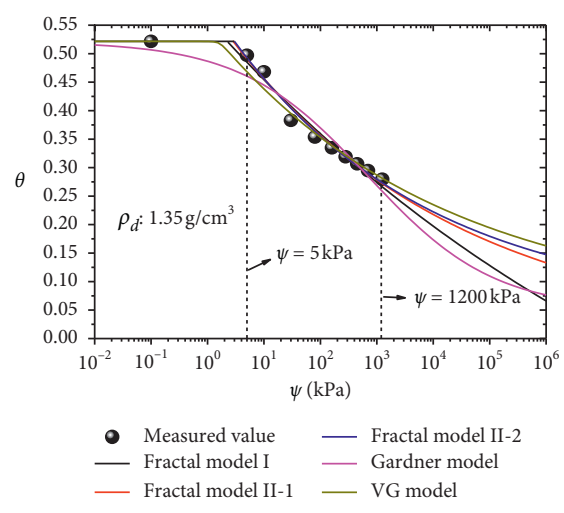

(b)

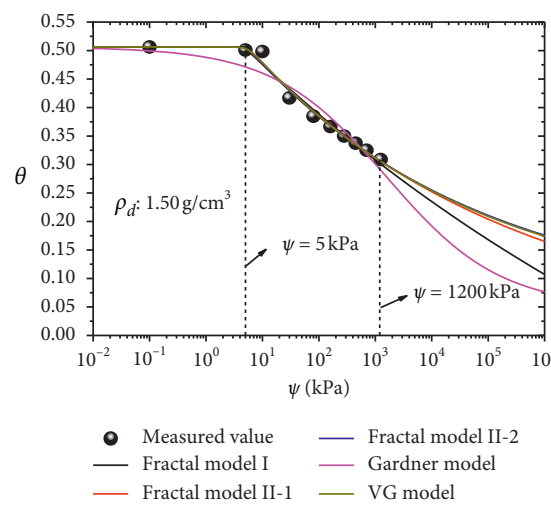

(e)

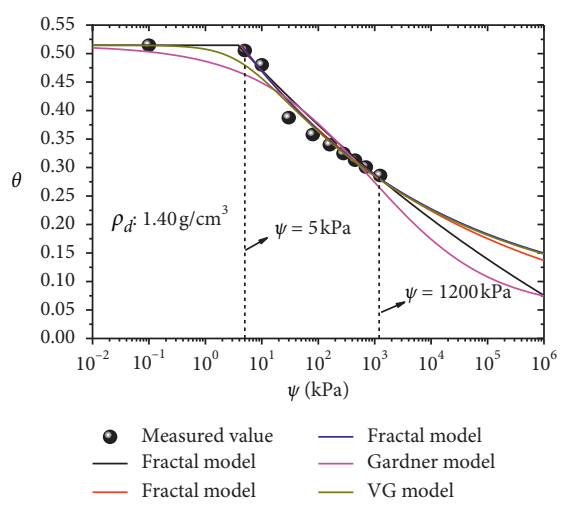

(c)

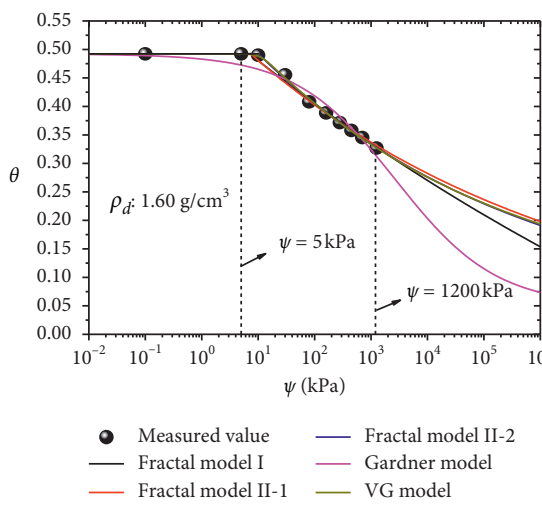

(f)

FIGURE 4: Fitting diagram of SWCC with initial different dry densities.

shows that the fractal model II-2 and the VG model are suitable for fitting the data obtained from the pressure plate instrument test. In addition, Gardner model fails to accurately characterize the relationship between volumetric water content and matrix suction in the low suction range. When matrix suction is in the range of $5 \sim 1200 \mathrm{kPa}$, the fitting results of the fractal and VG model have little difference. However, when the matrix suction reaches $1200 \mathrm{kPa}$, the fitting results of different models are significantly different. Five models fail to show a trend that water content will tend to zero as suction increases. Compared with the indoor evaporation experiment data, it is found that the SWCC of high suction cannot be predicted accurately due to the limit of test data. The values of $\theta_{r}$ obtained by fractal model I and Gardner model are in good agreement with the results of indoor evaporation test, but the fitting results of fractal model I are greatly effected by dry density. Note that the data points less than the air-entry value $\psi_{a}$ $\left(\psi>\psi_{a}\right)$ should be ignored in the fitting process of fractal model, while those data are considered for empirical model. A summary of data used is provided in Supplementary Tables S3-S8.

The correlation coefficients $R^{2}$ obtained from the fitting results of different SWCC models are shown in Table 3. It can be found that $R^{2}$ of the fractal model II- 2 and the VG model is the highest, the fractal model II- 1 and the fractal model I are the second, and the Gardner model is the lowest.
Meanwhile, the values of $R^{2}$ of fractal model II-2 are higher than those of II-1, indicating that the fitting accuracy of the fractal model can be improved effectively when $\theta_{r}$ is considered.

\subsection{Effect of Initial Dry Density on Model Parameters.} Table 4 shows the model parameter fitting values at different initial dry densities. It can be seen from Table 4 that the variation law of different model parameter values with the increase of initial dry density is quite different. For the Gardner model, the value of parameter $a$ decreases with the increase of the initial dry density, while the value of parameter $\lambda$ increases with the initial dry density increases. For the VG model, the fitting values of parameter $a$ and $m$ decrease with the increase of initial dry density and the value of parameter $p$ increases with the increase of initial dry density.

Table 5 shows the fitting values of fractal parameters at different initial dry densities, in which $D$ is fractal dimension and $\psi_{a}$ denotes the air-entry value $(\mathrm{kPa})$. From Table 5 , it can be found that $\psi_{a}$ increases as initial dry density increases, resulting from the fact that the larger the initial dry density is, the higher the soil compactness is and the harder the soil loses water at low suction. At the same initial dry density, the fitting values of $\psi_{a}$ have little difference in the three models. For the values of $D$, there are some differences of fitting results in different models. However, with the increase of 
TABLe 3: Correlation coefficients $R^{2}$ determined by model fitting.

\begin{tabular}{|c|c|c|c|c|c|}
\hline Dry density $\left(\mathrm{g} / \mathrm{cm}^{3}\right)$ & $\begin{array}{l}\text { Fractal model I } \\
R^{2}\end{array}$ & $\begin{array}{l}\text { Fractal model II-1 } \\
\qquad R^{2}\end{array}$ & $\begin{array}{l}\text { Fractal model II-2 } \\
\qquad R^{2}\end{array}$ & $\begin{array}{c}\text { Gardner's model } \\
R^{2}\end{array}$ & $\begin{array}{c}\text { VG model } \\
R^{2}\end{array}$ \\
\hline 1.30 & 0.9584 & 0.9736 & 0.9874 & 0.9384 & 0.9849 \\
\hline 1.35 & 0.9687 & 0.9702 & 0.9827 & 0.9361 & 0.9722 \\
\hline 1.40 & 0.9614 & 0.9640 & 0.9766 & 0.9173 & 0.9686 \\
\hline 1.45 & 0.9632 & 0.9710 & 0.9724 & 0.9190 & 0.9806 \\
\hline 1.50 & 0.9746 & 0.9798 & 0.9841 & 0.9641 & 0.9870 \\
\hline 1.60 & 0.9870 & 0.9829 & 0.9982 & 0.9488 & 0.9983 \\
\hline
\end{tabular}

Table 4: Model parameters value with different initial densities determined by fitting.

\begin{tabular}{lccccc}
\hline & \multicolumn{2}{c}{$\begin{array}{c}\text { Gardner's } \\
\text { model }\end{array}$} & \multicolumn{3}{c}{ VG model } \\
& $a$ & $\lambda$ & $a$ & $m$ & $p$ \\
\hline 1.30 & 0.1134 & 0.3622 & 1.2440 & 11.2600 & 0.0089 \\
1.35 & 0.0797 & 0.3874 & 0.3619 & 8.1920 & 0.0235 \\
1.40 & 0.0647 & 0.4069 & 0.2636 & 7.5150 & 0.0263 \\
1.45 & 0.0518 & 0.4184 & 0.2714 & 3.1110 & 0.0346 \\
1.50 & 0.0415 & 0.4318 & 0.1741 & 2.0780 & 0.0519 \\
1.60 & 0.0212 & 0.4878 & 0.0973 & 1.4790 & 0.0677 \\
\hline
\end{tabular}

initial dry density, the value of $D$ changes insignificantly, indicating that the initial dry density has little effect on $D$.

\subsection{The Effect of Residual Water Content on the Model Fitting.} The dependence of various models (fractal model II-2, Gardner's model, and VG model) on the limit range of $\theta_{r}$ is shown in Figure 5. $\theta_{r}$ is limited in $0<\theta_{r} \leq 5 \%$ and $\theta_{r}>0$, respectively. When matrix suction is less than $1200 \mathrm{kPa}$, the fitting results of the different models are the same and the limited range of $\theta_{r}$ has little effect on the fitting results. Meanwhile, the fitting accuracy of fractal model II-2 and VG model is better than that of Gardner's model. However, when the matrix suction is beyond $1200 \mathrm{kPa}$, the limited range of $\theta_{r}$ has significant influence on the fitting results, in which the fitting results of model with $\theta_{r}$ in the range of $0<\theta_{r} \leq 5 \%$ are better than those of model with $\theta_{r}$ in the range of $\theta_{r}>0$. Therefore, the reasonable limit range of $\theta_{r}$ can effectively improve the fitting accuracy in the high suction range $(\psi>1200 \mathrm{kPa})$. Among the three models analyzed, the Gardner model has the best prediction results for high suction range. A summary of data used is provided in Supplementary Tables S9-S14.

Table 6 shows the fitting and measured values of residual water content at different initial dry densities. $\theta_{r}$ in Table 6 denotes the measured value of residual water content. $\theta_{r 1}$ and $\theta_{r 2}$ are the fitting value of residual water content values and correspond to the fitting results of limited range and unlimited range, respectively. As can be seen from Table 6 , the error between $\theta_{r 2}$ and $\theta_{r}$ is greater than that between $\theta_{r 1}$ and $\theta_{r}$, but there is still a great difference between the values of $\theta_{r 1}$ and $\theta_{r}$. In addition, the measured $\theta_{r}$ increases with the increase of the initial dry density of the sample, while $\theta_{r 1}$ according to the model fitting remains basically unchanged, and there is no such
TABle 5: The fractal dimension $D$ and the air-entry value $\psi_{\mathrm{a}}$ determined by model fitting.

\begin{tabular}{lcccccc}
\hline $\begin{array}{l}\text { Dry density } \\
\left(\mathrm{g} / \mathrm{cm}^{3}\right)\end{array}$ & \multicolumn{2}{c}{ Fractal model I } & \multicolumn{2}{c}{$\begin{array}{c}\text { Fractal model II- } \\
1\end{array}$} & \multicolumn{2}{c}{ Fractal model } \\
& $D$ & $\psi_{a}(\mathrm{kPa})$ & $D$ & $\psi_{a}(\mathrm{kPa})$ & $D$ & $\psi_{a}(\mathrm{kPa})$ \\
\hline 1.30 & 2.952 & 1.502 & 2.887 & 2.102 & 2.869 & 2.256 \\
1.35 & 2.954 & 2.880 & 2.893 & 3.515 & 2.876 & 3.663 \\
1.40 & 2.955 & 6.103 & 2.893 & 6.678 & 2.876 & 6.789 \\
1.45 & 2.959 & 7.701 & 2.901 & 8.154 & 2.886 & 8.217 \\
1.50 & 2.961 & 16.280 & 2.908 & 15.850 & 2.894 & 15.710 \\
1.60 & 2.968 & 61.830 & 2.9230 & 57.230 & 2.910 & 64.640 \\
\hline
\end{tabular}

rule, which may be caused by the lack of high suction range measured with the model fitting.

In Figure 4, the limited range of $\theta_{r}$ has little effect on the model fitting when the matrix suction is less than the matrix suction of experimental data. When the matrix suction is beyond the maximum matrix suction of experimental data, the limited range of $\theta_{r}$ can significantly improve the fitting accuracy of model to a certain extent, but still has significant difference with actual situation. Meanwhile, at high suction range, the limited range of $\theta_{r}$ can only predict the general trend of SWCCs, it cannot accurately describe the relationship between the matrix suction and the water content. In conclusion, the prediction accuracy of models has great dependency on the range of experimental data.

4.4. Discussions. Based on experimental SWCCs, the fitting results of fractal models (fractal models I and II) and existing empirical models (Gardner's and VG model) are compared. It is found that the fitting results of fractal model II-2 and VG model are similar in the range of measured suction and have the best coincidence degree with the measured value, while that of Gardner model is the worst. During the full range of suction, when suction exceeds the measured value $(1200 \mathrm{kPa})$, the difference of fitting results of different models increases gradually with the increase of suction. However, due to the lack of measured data in the range of high suction section, the fitting effect in this range is hard to be accurately judged. Therefore, this paper indirectly evaluates the fitting effect of different models in high suction range by comparing the difference between the fitting and measured values of residual water content. Meanwhile, the influence of the limited range of residual water content on the fitting 


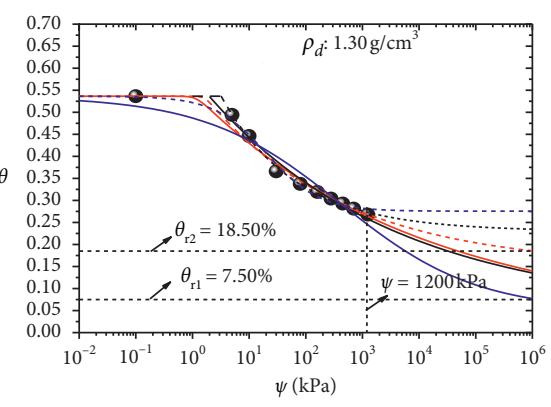

- Measured value

Fractal model II-2 — limited - - unlimited

Gardner model — limited - - unlimited

VG model _ limited - - unlimited

(a)

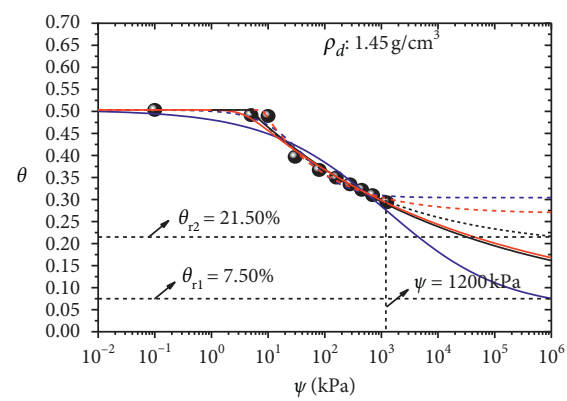

- Measured value

Fractal model II-2 — limited - - unlimited

Gardner model _ limited - - unlimited

VG model _ limited - - unlimited

(d)

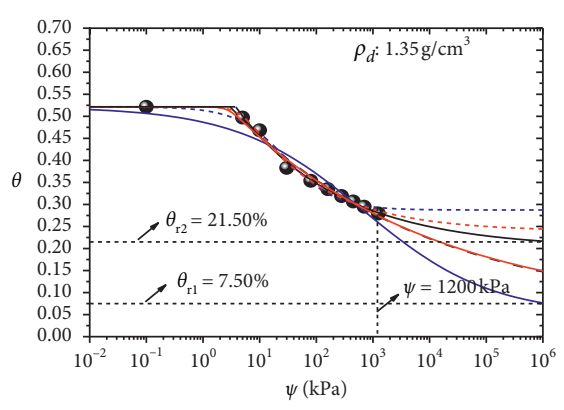

- Measured value

Fractal model II-2 _ limited - - unlimited

Gardner model _ limited -- unlimited

VG model $\quad$ - limited -- unlimited

(b)
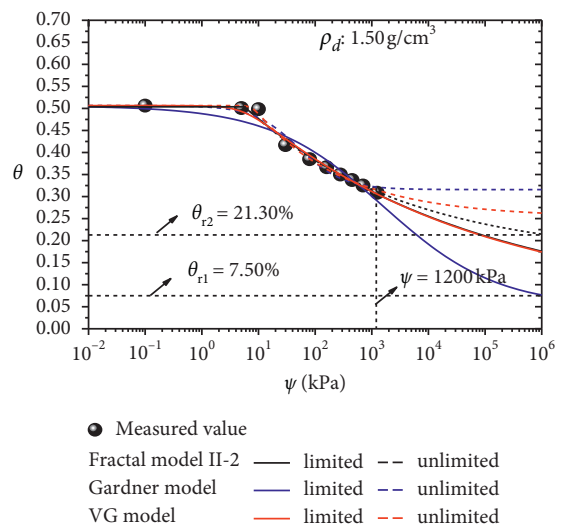

(e)

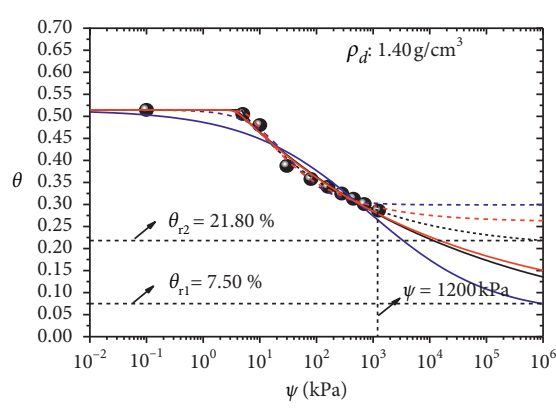

- Measured value

Fractal model II-2 — limited - - unlimited

Gardner model _ limited -- unlimited

VG model _ l limited -- unlimited

(c)

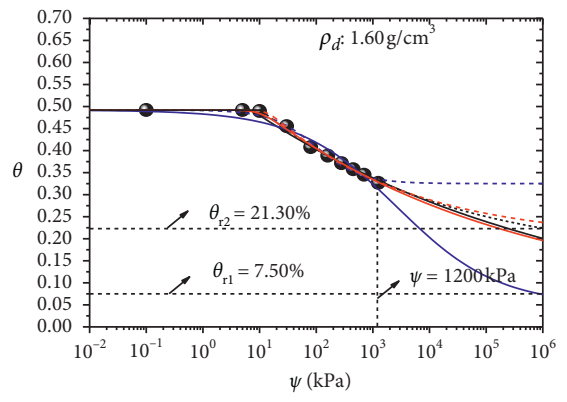

- Measured value

Fractal model II-2 — limited -- unlimited

Gardner model _ limited -- unlimited

VG model $\quad$ limited -- unlimited

(f)

FIGURE 5: Fitting comparison diagram of limited and unlimited residual water content models.

TABLE 6: The fitting value and the measured value of residual water content $\theta_{r}$.

\begin{tabular}{lccccccc}
\hline & \multicolumn{2}{c}{ Gardner's model } & \multicolumn{2}{c}{ VG model } & \multicolumn{2}{c}{ Fractal model II-2 } \\
Dry density $\left(\mathrm{g} / \mathrm{cm}^{3}\right)$ & $0<\theta_{r} \leq 5 \%$ & $\theta_{r}>0$ & $0<\theta_{r} \leq 5 \%$ & $\theta_{r}>0$ & $0<\theta_{r} \leq 5 \%$ & $\theta_{r}>0$ & Measured value \\
& $\theta_{r 1}(\%)$ & $\theta_{r 2}(\%)$ & $\theta_{r 1}(\%)$ & $\theta_{r 2}(\%)$ & $\theta_{r 1}(\%)$ & $\theta_{r 2}(\%)$ & $\theta_{r}(\%)$ \\
\hline 1.30 & 7.50 & 27.60 & 14.10 & 18.70 & 13.80 & 23.30 & 2.90 \\
1.35 & 7.50 & 29.00 & 15.00 & 24.20 & 15.00 & 21.50 & 3.90 \\
1.40 & 7.50 & 29.90 & 15.00 & 26.20 & 13.50 & 21.80 & 4.10 \\
1.45 & 7.50 & 30.50 & 17.00 & 27.00 & 16.00 & 21.50 & 4.30 \\
1.50 & 7.50 & 31.90 & 17.60 & 26.10 & 17.80 & 21.30 & 4.50 \\
1.60 & 7.50 & 32.50 & 19.80 & 23.80 & 20.00 & 22.30 & 4.80 \\
\hline
\end{tabular}

accuracy is analyzed (see Figure 5). The results show that the fitting results of different models from limited test data in high suction range are far from the actual situation, and the limitation of the residual water content range can only improve the fitting accuracy in high suction range to a certain extent. Therefore, it is suggested that the measured data in high suction range should be appropriately added when it is necessary to obtain the accurate fitting curve over full suction range (especially the high suction range). In addition, compared with empirical models, fractal models contain less parameters with obvious physical significance, and much workload can be reduced in the fitting process.

\section{Conclusion}

This paper discusses the difference between two kinds of fractal models and the existing empirical models (Gardner's and VG model) in fitting SWCC over full suction range. The main conclusions are presented as follows:

(1) Based on limited experimental SWCCs of Hunan clay, the fitting results of different models show that when the suction is in the range of measured data, the fitting results of five models have little difference, where fractal model II-2 and VG model are the best, followed by the fractal models I and II-1, and those of the Gardner model are the worst. When the suction is beyond the range of 
measured data, the difference of fitting results of different models increases gradually as suction increases.

(2) As for the model with parameter $\theta_{r}$, the limit of residual water content range can only improve the fitting accuracy in high suction range to a certain extent. If an accurate fitting curve over whole suction range (especially the high suction range) is demanded, more experimental SWCCs of high suction should be appropriately added.

(3) The $D$ and $\psi_{a}$ values obtained by fractal model show that there is some difference in $D$ between different models. In addition, the initial dry density has insignificant effect on $D$ but has an obvious effect on $\psi_{a}$; its value increases with the increase of the initial dry density.

\section{Data Availability}

The data used to support the findings of this study are available from the corresponding author upon request.

\section{Conflicts of Interest}

The authors declare that there are no conflicts of interest regarding the publication of this paper.

\section{Acknowledgments}

The research was funded by the National Natural Science Foundation of China (Grant number 51978249) and Outstanding Young and Middle-Aged Scientific and Technological Innovation Team Project of Hubei Provincial Education Department (Grant number T201605).

\section{Supplementary Materials}

S1: this table is corresponding to Figure 2 (measured values of SWCCs of Hunan clay with different initial dry densities). S2: this table is corresponding to Figure 3 (variation curve of mass water content of Hunan clay with time). S3: this table is corresponding to Figure 4(a) (fitting diagram of SWCCs with initial different dry densities). S4: this table is corresponding to Figure 4(b) (fitting diagram of SWCCs with initial different dry densities). S5: this table is corresponding to Figure 4(c) (fitting diagram of SWCCs with initial different dry densities). S6: this table is corresponding to Figure 4(d) (fitting diagram of SWCCs with initial different dry densities). S7: this table is corresponding to Figure 4(e) (fitting diagram of SWCCs with initial different dry densities). S8: this table is corresponding to Figure 4(f) (fitting diagram of SWCCs with initial different dry densities). S9: this table is corresponding to Figure 5(a) (fitting comparison diagram of limited and unlimited residual water content models). S10: this table is corresponding to Figure 5(b) (fitting comparison diagram of limited and unlimited residual water content models). S11: this table is corresponding to Figure 5(c) (fitting comparison diagram of limited and unlimited residual water content models). S12: this table is corresponding to Figure 5(d) (fitting comparison diagram of limited and unlimited residual water content models). S13: this table is corresponding to Figure 5(e) (fitting comparison diagram of limited and unlimited residual water content models). S14: this table is corresponding to Figure 5(f) (fitting comparison diagram of limited and unlimited residual water content models). (Supplementary Materials)

\section{References}

[1] S. M. Wang, D. F. Liu, Y. Z. Zhi, and X. P. Li, "Experimental research on soil-water characteristics curves of soils for a landslide," Rock and Soil Mechanics, vol. 29, no. 10, pp. 2651-2654, 2008.

[2] B. C. Zhou, L. W. Kong, W. Chen, H. Bai, and X. G. Li, "Anyalsis of characteristic parameters of soil-water characteristic cuver (SWCC) and unsaturated shear strength prediction of JingMen expansive soil," Chinese Journal of Rock Mechanics and Engineering, vol. 29, no. 5, pp. 1052-1059, 2010.

[3] X. D. Liu and J. Y. Shi, "Unsaturated conductivity of MSW based on soil-water characteristic curve," Chinese Journal of Rock Mechanics and Engineering, vol. 34, no. 5, pp. 855-862, 2012.

[4] Z. H. Chen, "On basic theories of unsaturated soils and special soils," Chinese Journal of Rock Mechanics and Engineering, vol. 36, no. 2, 2013.

[5] G. L. Tao, X. L. Zhu, J. C. Cai, H. L. Xiao, Q. S. Chen, and Y. Chen, "A fractal approach for predicting unsaturated hydraulic conductivity of deformable clay," Geofluids, vol. 2019, Article ID 8013851, 9 pages, 2019.

[6] W. R. Gardner, "Some steady-state solutions of the unsaturated moisture flow equation with application to evaporation from a water table," Soil Science, vol. 85, no. 4, pp. 228-232, 1958.

[7] M. T. van Genuchten, "A closed-form equation for predicting the hydraulic conductivity of unsaturated soils," Soil Science Society of America Journal, vol. 44, no. 5, pp. 892-898, 1980.

[8] D. G. Fredlund and A. Xing, "Equations for the soil-water characteristic curve," Canadian Geotechnical Journal, vol. 31, no. 4, pp. 521-532, 1994.

[9] D. Gallipoli, S. J. Wheeler, and M. Karstunen, "Modelling the variation of degree of saturation in a deformable unsaturated soil," Géotechnique, vol. 53, no. 53, pp. 105-112, 2003.

[10] H. Q. Pham, D. G. Fredlund, and S. L. Barbour, "A practical hysteresis model for the soil-water characteristic curve for soils with negligible volume change," Géotechnique, vol. 53, no. 2, pp. 293-298, 2003.

[11] C. T. Omuto, "Biexponential model for water retention characteristics," Geoderma, vol. 149, pp. 0-242, 2009.

[12] Z. Zhang, F. Y. Liu, X. G. Zhao, and D. Zhou, "A soil water characteristic curve model considering void ratio variation with stress," Journal of Hydraulic Engineering, vol. 39, 2013.

[13] A. Ahangar-Asr, A. Johari, and A. A. Javadi, "An evolutionary approach to modelling the soil-water characteristic curve in unsaturated soils," Computers \& Geosciences, vol. 43, pp. 25-33, 2012.

[14] Q. D. Huang, C. G. Zhao, and G. Q. Cai, "A thermodynamicsbased soil-water characteristic model with considering volumetric change and hysteresis," Rock and Soil Mechanics, vol. 37, no. 7, pp. 1857-1867, 2016.

[15] S. J. Wang, S. M. Cheng, X. Li, X. Q. Wang, X. Mao, and H. D. Yang, "Establishment of generalized soil-water characteristic curve theoretical model considering two stress state variables for unsaturated soils," Transactions of the Chinese Society of Agricultural Engineering, vol. 6, pp. 8-14, 2017. 
[16] H. Q. Pham and D. G. Fredlund, "Equations for the entire soil-water characteristic curve of a volume change soil," Canadian Geotechnical Journal, vol. 45, no. 4, pp. 443-453, 2008.

[17] S. Roy and S. Rajesh, "Influence of confining pressure on water retention characteristics of compacted soil," Indian Geotechnical Journal, vol. 48, no. 2, pp. 327-341, 2018.

[18] X. Ren, J. Kang, J. Ren, X. Chen, and M. Zhang, "A method for estimating soil water characteristic curve with limited experimental data," Geoderma, vol. 360, Article ID 114013, 2020.

[19] Q. Zhai, H. Rahardjo, and A. Satyanaga, "Effects of residual suction and residual water content on the estimation of permeability function," Geoderma, vol. 303, pp. 165-177, 2017.

[20] S. Rajesh, S. Roy, and S. Madhav, "Study of measured and fitted SWCC accounting the irregularity in the measured dataset," International Journal of Geotechnical Engineering, vol. 11, no. 4, pp. 321-331, 2017.

[21] A. J. Puppala, K. Punthutaecha, and S. K. Vanapalli, "Soilwater characteristic curves of stabilized expansive soils," Journal of Geotechnical and Geoenvironmental Engineering, vol. 132, no. 6, pp. 736-751, 2006.

[22] W. S. Kim and R. H. Borden, "Influence of soil type and stress state on predicting shear strength of unsaturated soils using the soil-water characteristic curve," Canadian Geotechnical Journal, vol. 48, no. 12, pp. 1886-1900, 2011.

[23] D. X. Chen and X. N. Gong, "Experiment and modeling of soil-water characteristic curve of unsaturated residual soil," Rock and Soil Mechanics, vol. 7, 2014.

[24] Y. Zhang, W. M. Ye, Q. Wng, and A. Tarantino, "Suction measurement and SWRC modelling for reconstituted saltladen soils in earthen heritages," Chinese Journal of Geotechnical Engineering, vol. 41, no. 9, 2018.

[25] B. Mandelbrot, "How long is the coast of Britain? Statistical self-similarity and fractional dimension," Science, vol. 156, no. 3775 , pp. 636-638, 1967.

[26] G. Huang and R. Zhang, "Evaluation of soil water retention curve with the pore-solid fractal model," Geoderma, vol. 127, p. 61, 2005.

[27] G. Tao, Y. Chen, L. Kong, H. Xiao, Q. Chen, and Y. Xia, “A simple fractal-based model for soil-water characteristic curves incorporating effects of initial void ratios," Energies, vol. 11, no. 6, p. 1419, 2018.

[28] D. L. Turcotte, "Fractals and fragmentation," Journal of Geophysical Research, vol. 91, no. B2, pp. 1921-1926, 1986.

[29] D. L. Turcotte, "Fractals in geology and geophysics," Fractals in Geophysics, vol. 131, no. 1-2, pp. 171-196, 1989.

[30] G. L. Tao, X. K. Wu, H. L. Xiao, Q. S. Chen, and G. H. Cai, “A unified fractal model for permeability coefficient of unsaturated soil," Fractals, vol. 27, no. 1, 2019.

[31] G. L. Tao and J. R. Zhang, "The fractal models of two types of rock-soil bodies characterized by pore size and particle volume and scale distribution," Chinese Science Bulletin, vol. 54, no. 6, pp. 838-846, 2009.

[32] M. A. A. Soto and H. K. Chang, "Fractal-based models for the unsaturated soil hydraulic functions," Geoderma, vol. 306, pp. 144-151, 2017.

[33] G. L. Gao, Y. Chen, H. L. Xiao, Q. S. Chen, and J. Wan, "Determining soil-water characteristic curves from mercury intrusion porosimeter test data using fractal theory," Energies, vol. 12, no. 4, pp. 752-767, 2019.

[34] S. W. Tyler and S. W. Wheatcraft, "Fractal processes in soil water retention," Water Resources Research, vol. 26, no. 5, pp. 1047-1054, 1990.
[35] M. Rieu and G. Sposito, "Fractal fragmentation, soil porosity, and soil water properties: I. Theory," Soil Science Society of America Journal, vol. 55, no. 5, pp. 1231-1238, 1991.

[36] E. Perfect, N. B. Mclaughlin, B. D. Kay, and G. C. Topp, "An improved fractal equation for the soil water retention curve," Water Resources Research, vol. 32, no. 2, pp. 281-287, 1996.

[37] E. Perrier, N. Bird, and M. Rieu, "Generalizing the fractal model of soil structure: the pore-solid fractal approach: the pore-solid fractal approach," Geoderma, vol. 88, no. 3-4, pp. 137-164, 1999.

[38] N. R. A. Bird, E. Perrier, and M. Rieu, "The water retention function for a model of soil structure with pore and solid fractal distributions," European Journal of Soil Science, vol. 51, no. 1, pp. 55-63, 2000.

[39] Y. Xu and D. A. Sun, "Determination of expansive soil strength using a fractal model," Fractals, vol. 9, no. 1, pp. 51-60, 2001.

[40] Y. F. Xu and P. Dung, "Fractal models soil-water characteristic curve of unsaturated soil," Rock and Soil Mechanics, vol. 23, no. 4, pp. 400-405, 2002.

[41] J. W. Crawford, N. Matsui, and I. M. Young, "The relation between the moisture-release curve and the structure of soil," European Journal of Soil Science, vol. 46, no. 3, pp. 369-375, 2010.

[42] G. L. Tao, J. R. Zhang, X. S. Zhuang, and L. Yang, “A fractal model describing the relation between clay content and soilwater characteristic curve," Journal of Hydraulic Engineering, vol. 39, no. Z2, 2014.

[43] M. H. Yang, H. Chen, and K. Chen, "Study of the hysteresis effect model of SWCC boundary curves based on fractal theory," Rock and Soil Mechanics, vol. 40, no. 10, 2019.

[44] J. R. Zhang, G. L. Tao, L. Huang, and L. Yuan, "The model and application of the porosity of the rock-soil with the pore size distribution," Chinese Science Bulletin, vol. 55, no. 34, pp. 2761-2770, 2010. 